\title{
On the relationship between gastrin, gastric secretion, and adjuvant arthritis in rats
}

\author{
PATRICK J. ROONEY*, W. CARSON DICK*, ROBERT C. IMRIE, \\ DAVID TURNER $\dagger$, KEITH D. BUCHANAN $\dagger$, AND JOY ARDILL +
}

SUMMARY The elevation of plasma immunoreactive gastrin known to occur during the induction of adjuvant-induced arthritis (Rooney et al., 1973) has been shown to be maximal at 7 days after injection. Gastrin administered exogenously accelerated and exacerbated the inflammatory joint disease. Some evidence has been presented that the endogenous immunoreactive gastrin had biological activity in terms of gastric acid secretion.

Previous studies having indicated significant elevation of plasma immunoreactive gastrin occurring in association with inflammatory joint disease both in human rheumatoid arthritis (Rooney et al., 1976) and in rats with adjuvant-induced arthritis (Rooney et al., 1973). The current study was undertaken to determine the relationship of changes in immunoreactive gastrin to the time course of adjuvant disease and to determine whether these changes in immunoreactive hormone were biologically meaningful in terms of gastric acid output. The effect of exogenously administered synthetic gastrin pentapeptide on the time course and severity of adjuvant disease was also studied.

\section{Materia's and methods}

The animals used in these studies were adult SpragueDawley rats each weighing around $300 \mathrm{~g}$. In all instances induction of adjuvant arthritis was by a standard procedure involving the sub-plantar injection of killed Mycobacterium butyricum in heavy mineral oil into the left hind paw. The standard dose was $0.5 \mathrm{mg}$ in $0.1 \mathrm{ml}$ oil, alterations to the dose are discussed below in detail. The progress and severity of the adjuvant disease was assessed by means of weight change and paw scores (Currey and Ziff, 1966) immediately before withdrawal of blood.

Accepted for publication 12 December, 1977

Correspondence to Dr J. Rooney, Floor 7, Gartnavel General Hospital, Great Western Road, Glasgow.

*The Centre for Rheumatic Diseases and University Department of Medicine, Royal Infirmary and the Glasgow Western District Teaching Complex.

†Beechams Laboratories, Betchworth, Surrey and Harlow Essex.

$\ddagger$ Department of Medicine, Queen’s University, Belfast.
Gastric fistula rats were prepared surgically least 2 weeks before study. The surgery consisted of the formation of a simple gastric fistula at th $\vec{B}$ juction of the main stomach and the rumen. Patencob was maintained by means of a titanium steet cannula.

Plasma gastrin was assayed in blood obtained fror the rat tail vein. In every instance the rats had beeg fasted for 15 hours overnight in wire mesh cages designed to prevent coprophagia. The hormone was measured by a sensitive and specific radioimmuno? assay. In this assay, the anti-serum is raised in rabbits to synthetic human gastrin 1 (I.C.I.). Labelled hot mone is prepared using a modification of the chloramine-T method of Hunter and Greenwoos (1962) and separation of free hormone from that bound to antibody is achieved by treatment of the assay incubate with dextran-coated charcoal (Buch anan and McCarroll, 1971). Precision is greatese below $600 \mathrm{pg} / \mathrm{ml}(\mathrm{ng} / \mathrm{ml})$ and the sensitivity of the assay, that is the lowest concentration of hormons that can be reliably differentiated from zero, is 19 $\mathrm{pg} / \mathrm{ml}(\mathrm{ng} / \mathrm{ml})$. Cross reaction with cholecystokinino pancreozymin is minimal and preliminary studies show that the antibody recognises not only the heptadecapeptide but also the 'big' gastrin described by Yalow and Berson (1970).

In the first group of 8 intact rats studied, the standard dose of adjuvant was used. These animats were fully assessed on days $0,1,7,14$, and 21 after injection. A further 3 groups, each of 16 intact animals, were injected with varying doses of adjuvan? In 1 , the standard dosage regime was used (thi would be expected to induce arthritis in $90 \%$ of animals). In the second group $0.3 \mathrm{mg} M$. butyricum was used and in the third $0.1 \mathrm{mg}$ each in the 
standard volume of oil. In addition in all 3 groups each rat received $5 \mu \mathrm{g}$ synthetic pentagastrin (I.C.I.) subcutaneously twice daily for 3 weeks following the injection of adjuvant. Paw scores were assessed on days, 7, 14, and 21 after the injection. Gastric acid output was assessed by both volume and concentration over a period of 30 minutes. The titration was carried out against sodium hydroxide using a $\mathrm{pH}$ meter.

\section{Results}

The time course of the elevation of plasma immunoreactive gastrin following the induction of adjuvant arthritis is shown by Table 1 and Fig. 1. The peak level of immunoreactive gastrin is reached on day 7 . This coincides with the onset of the secondary

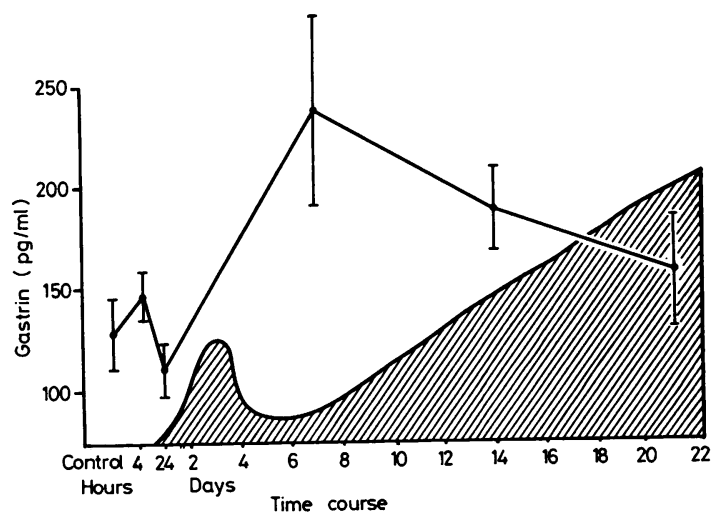

Fig. 1 Gastrin in adjuvant arthritis. The elevation of mean ( \pm SEM) immunoreactive gastrin in rats at 7 days is shown by the black line. The hatched area indicates the course of the inflammatory joint disease with the small initial rise due to the inflammation locally in the injected paw followed by the progressive generalised arthropathy. inflammatory response to the injected paw. Thereafter as the generalised arthropathy develops immunoreactive gastrin concentration falls.

The effect of exogenously injected gastrin on the course of adjuvant-induced arthritis is shown in Table 2 and Fig. 2. These data indicate that the exhibition of pentagastrin in this way causes earlier onset of generalised joint disease and that this occurs

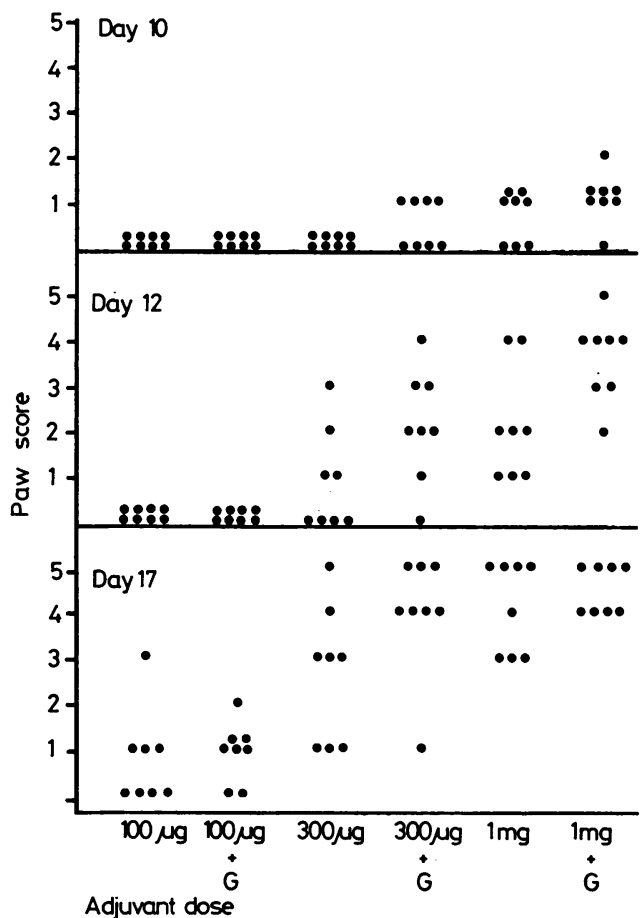

Fig. 2 Paw scores in rats receiving sub-optional doses of adjuvant. In each group the rats also receiving gastrin injections $(+G)$ are compared to those receiving adjuvant alone.

Table 1 Immunoreactive gastrin ( $\mathrm{pg} / \mathrm{ml} ; \mathrm{ng} / \mathrm{ml})$ in intact rats on the day before and on days $1,7,14$, and 21 following injection of standard adjuvant

\begin{tabular}{|c|c|c|c|c|c|}
\hline \multirow[t]{2}{*}{ Rat } & \multicolumn{5}{|l|}{ Day } \\
\hline & 0 & 1 & 7 & 14 & 21 \\
\hline $\begin{array}{l}1 \\
2 \\
3 \\
4 \\
5 \\
6 \\
7 \\
8\end{array}$ & $\begin{array}{r}60 \\
115 \\
165 \\
145 \\
210 \\
75 \\
135 \\
125\end{array}$ & $\begin{array}{r}85 \\
155 \\
125 \\
135 \\
125 \\
65 \\
85 \\
-\end{array}$ & $\begin{array}{l}105 \\
155 \\
230 \\
495 \\
240 \\
230 \\
210\end{array}$ & $\begin{array}{l}250 \\
165 \\
165 \\
135 \\
105 \\
180 \\
250 \\
260\end{array}$ & $\begin{array}{r}105 \\
180 \\
50 \\
135 \\
260 \\
80 \\
145 \\
230\end{array}$ \\
\hline Mean \pm SEM & $128 \cdot 7 \pm 16 \cdot 9$ & $110 \cdot 7 \pm 12 \cdot 3$ & $237 \cdot 8 \pm 46 \cdot 7$ & $188 \cdot 7 \pm 20 \cdot 3$ & $157 \cdot 8 \pm 27 \cdot 3$ \\
\hline
\end{tabular}


Table 2 Paw scores in rats receiving varying doses of adjuvant and concomitant administration of pentagastrin (I.C.I.) in a dose of $5 \mathrm{pg}$ per rat twice daily

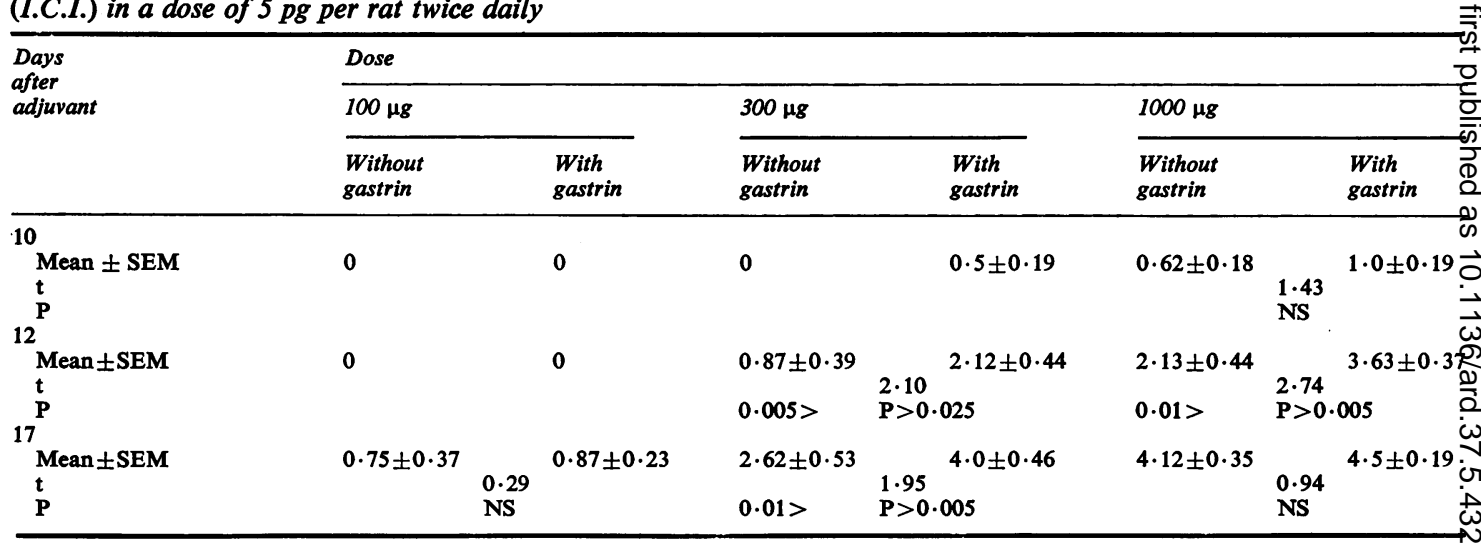

at lower doses of injected adjuvant than is usually required, except in those rats receiving $100 \mathrm{~g}$ of the adjuvant. The mean paw scores at $\mathbf{1 2}$ days in those rats not receiving gastrin were significantly lower than those in the injected animals where both the full adjuvant dose as well as the $0.3 \mathrm{mg}$ dose had been given. A similar effect was seen in those rats receiving $0.3 \mathrm{mg}$ adjuvant at 17 days but in view of the severity of disease in both the pentagastrin-treated and untreated groups this effect is no longer apparent in those rats given full doses of adjuvant.

The effect of adjuvant-induced arthritis on the basal gastric acid secretion is summarised in Table 3. It is clear that at 7 days after adjuvant injection fall in gastric acid output occurred. However, although some rise in immunoreactive gastrin is noted at this time in the fistula rats, this failed to reach levels of statistical significance. It may be significant that the severity of the arthritis in the rats with fistulae was much less than that observed in the other experiments although the weight changes expected to accompany the inflammatory joint diseases were still readily apparent.

\section{Discussion}

Significant elevation of fasting immunoreactive gastrin occurs during the induction of adjuvant arthritis (Rooney et al., 1973). The mechanism of this effect is unclear, but if it reflects the samed phenomenon as has been reported in some patients with rheumatoid arthritis (Rooney et al., 1976), i⿱ is clear that drug therapy and specific rheumatoi factors are not implicated in its production. It seemß likely that it will prove to be part of the cascade oक phenomena associated with the inflammatory responses (McQueen, 1973; Ward, 1974). Certainle in these studies the peak increase in the concentration of plasma immunoreactive gastrin coincides wit the severe inflammatory disease characteristically induced by adjuvant injection (Pearson and Wood 1963).

The action of gastrin in this model appears to be a phlogistic one as evidenced by the effect of exos genous gastrin on the onset and severity of join disease. This finding suggests that gastrin is eitheg an active mediator of inflammation or that it secretion parallels that of another agent with this

Table 3 Mean values ( $\pm S E M)$ for weight volume of gastric juice, total titratable activity (TTA), and plasma immunoreactive gastrin concentration in rats with gastric fistulae after induction of adjuvant arthritis

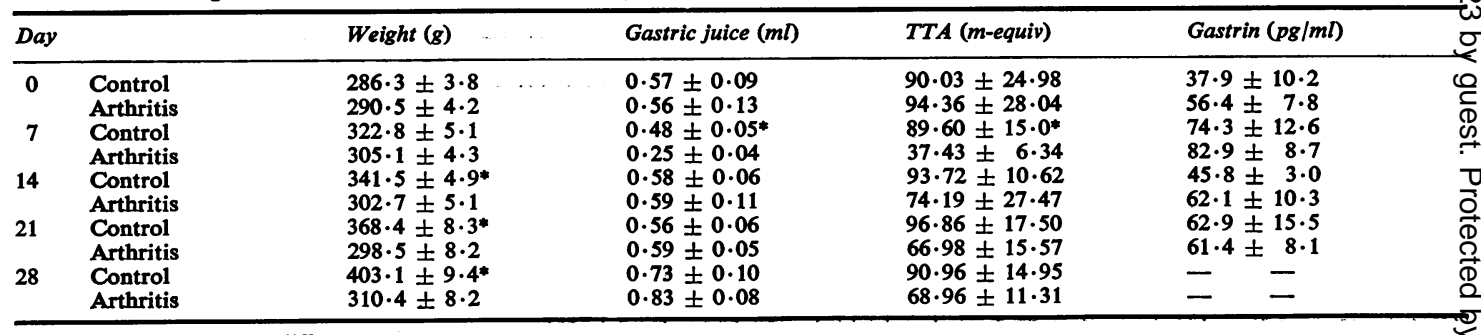

* $t$ Test shows significant difference. 
effect. In the human disease situation, the absence of the normal biological effect of the hormone, the secretion of gastric acid, in the face of a normal target organ (Rooney et al., 1976) tends to suggest that gastrin may not be the primary agent in the effect. Prostaglandins are known to be involved in inflammatory responses (Greaves et al., 1971) and prostaglandin $\mathrm{E}_{2}$ and its methylated analogues have been shown to inhibit gastric acid secretion whether the stimulus to this be gastrin, histamine, or vagal activity (Karim et al., 1973). It is conceivable, therefore, that the involvement of prostaglandins or related mediators in the inflammatory cascade results in inhibition of gastric acid secretion with resultant biofeedback leading to hypergastrinaemia as occurs in atrophic gastritis (Ganguli et al., 1971).

It is interesting, in these experiments, that, although some biological effects on the target organ for gastrin were observed, in that changes in gastric secretion occurred in the fistula rats, no marked elevation of plasma immunoreactive gastrin was observed. Whether this reflects the reduction in the severity of the arthropathy observed or whether the presence of the fistula interferes in some way with the stimulus to gastrin secretion is not known. It is also interesting to speculate whether the presence of this fistula contributed in some way to protecting the animals from the inflammatory joint disease.

\section{References}

Buchanan, K. D., and McCarroll, A. M. (1971). Separation techniques. Radioimmunoassay Methods, ed. by K. E. Kirkham and W. M. Hunter, pp. 136-148. Churchill Livingstone, London.
Currey, H. L. F., and Ziff, M. (1966). Suppression of experimentally induced polyarthritis in the rat by heterologous anti-lymphocyte serum. Lancet, 2, 889-891.

Ganguli, P. C., Cullen, D. R., and Imrie, W. J. (19.71). Radioimmunoassay of plasma gastrin in pernicious anaemia, achlorhydria without pernicious anaemia, hypochlorhydria and controls. Lancet, 1, 155-158.

Greaves, M. W., Sondergaard, J., and McDonald Gibson, W. (1971). Recovery of prostaglandins in human cutaneous inflammation. British Medical Journal, 2, 258-260.

Hunter, W. M., and Greenwood, F. C. (1962). Preparation of iodine ${ }^{131}$ labelled human growth hormone of high specific activity. Nature, 194, 495-496.

Karim, S. M. M., Carter, D. C., Bhana, D., and AdaikanGaneson, P. (1973). Effect of orally administered prostaglandin $E_{2}$ and its 15 methyl analogues on gastric secretion. British Medical Journal, 1, 143-146.

McQueen, E. G. (1973). Anti-inflammatory drug mechanisms. Drugs, 6, 104-117.

Pearson, C. M., and Wood, F. D. (1963). Studies of arthritis and other lesions induced in rats by the injection of mycobacterial adjuvant. VII. Pathologic details of the arthritis and spondylitis. American Journal of Pathology, 42, 723-735.

Rooney, P. J., Dick, W. C., Buchanan, K. D., and Imrie, R. C. (1973). An animal model for the study of the relationship between immuno-reactive gastrin and inflammatory arthritis. Nature, 246, 497-498.

Rooney, P. J., Kennedy, A. C., Gray, G. H., Sturrock, R. D., Buchanan, K. D., and Dick, W. C. (1976). Serum immunoreactive gastrin (IRG) in rheumatoid arthritis: further observations on its identity, clinical specificity and relationship to gastric function. Annals of the Rheumatic Diseases, 35, 246-250.

Ward, P. A. (1974). The inflammatory mediators. Annals of the New York Academy of Sciences, 221, 290-298.

Y̌alow, R.'S., and Berson, S. A. (1970). Radio-immunoassay of gastrin. Gastroenterology, 58, 1-14. 